VIRAL HEPATITIS

\title{
Peginterferon $\alpha-2 b$ and ribavirin therapy in chronic hepatitis C genotype 4: impact of treatment duration and viral kinetics on sustained virological response
}

\author{
S M Kamal, A A El Tawil, T Nakano, Q He, J Rasenack, S A Hakam, W A Saleh, A Ismail, A A Aziz, \\ M Ali Madwar
}

Gut 2005;54:858-866. doi: 10.1136/gut.2004.057182

See end of article for authors' affiliations

Correspondence to: Dr S M Kamal, Department of Gastroenterology and Liver Disease, Ain Shams Faculty of Medicine, $22 \mathrm{Al}$ Ahram St, Roxy,

Heliopolis, Cairo, Egypt; sanaa.kamal@link.net

Received

24 September 2004

Accepted for publication

26 November 2004
Background: The response rates and duration of peginterferon alpha (PEG-IFN- $\alpha$ ) and ribavirin combination therapy in chronic hepatitis $\mathrm{C}$ genotype 4, the prevalent genotype in the Middle East and Africa, are poorly documented.

Aims: To compare the efficacy and safety of 24,36 , or 48 weeks of PEG-IFN- $\alpha-2 b$ and ribavirin therapy in chronic hepatitis $C$ genotype 4 .

Methods: In this prospective, randomised, double blind study, 287 patients with chronic hepatitis C genotype 4 were randomly assigned to PEG-IFN- $\alpha-2 b(1.5 \mu \mathrm{g} / \mathrm{kg})$ once weekly plus daily ribavirin (1000-1200 mg) for 24 weeks (group A, $n=95$ ), 36 weeks (group B, $n=96$ ), or 48 weeks (group C, $\mathrm{n}=96$ ) and followed for 48 weeks after completion of treatment. Early viral kinetics and histopathological evaluation of pre- and post treatment liver biopsies were performed. The primary end point was viral clearance 48 weeks after completion of treatment.

Results: Sustained virological response was achieved in $29 \%, 66 \%$, and $69 \%$ of patients treated with PEGIFN- $\alpha-2 b$ and ribavirin for 24,36 , and 48 weeks, respectively, by intention to treat analysis. No statistically significant difference in sustained virological response rates was detected between 36 and 48 weeks of therapy $(p=0.3)$. Subjects with sustained virological response showed greater antiviral efficacy $(\epsilon)$ and rapid viral load decline from baseline to treatment week 4 compared with non-responders and improvement in liver histology. The incidence of adverse events was higher in the group treated for 48 weeks.

Conclusion: PEG-IFN- $\alpha-2 b$ and ribavirin for 36 or 48 weeks was more effective in the treatment of chronic hepatitis $C$ genotype 4 than treatment for 24 weeks. Thirty six week therapy was well tolerated and produced sustained virological and histological response rates similar to the 48 week regimen.
$\mathrm{T}$ he WHO has estimated that 170 million people worldwide are infected with hepatitis C virus (HCV). Reports indicate that the prevalence rates for anti-HCV ranges from $0.5-1 \%$ in low prevalence areas such as North America to $>20 \%$ in high prevalence areas such as Egypt. ${ }^{12}$ Acute infection with HCV is followed by chronic infection in $80 \%$ of cases with increased risk for liver cirrhosis and hepatocellular carcinoma. $^{3}{ }^{4}$

HCV genotype differences are of considerable clinical significance because they affect responses to antiviral therapy. Genotype 4 predominates throughout the Middle East and parts of Africa, ${ }^{15}$ often in association with a high population prevalence as in Egypt, ${ }^{6-8}$ and exists to a lesser extent in other areas, such as southern Europe and the USA. ${ }^{10}$ More than $90 \%$ of Egyptian HCV isolates belong to genotype $4 .^{5-8}$ Phylogenetic analysis of the complete genomic sequence of genotype 4 revealed a closer relationship between genotype 4 and genotype 1 than with other genotypes, as evidenced by the short branch and slightly greater degree of nucleotide sequence similarity between genotypes 1 and 4 than that found between other genotypes. ${ }^{511}$ Subtype $4 a$ is the dominant Egyptian HCV strain but other subtypes (provisionally named $4 \alpha, 4 \beta$, and $\lg$ ) are also present at a lower prevalence rate. ${ }^{12}$

Earlier reports showed that patients infected with HCV genotype 4 (HCV-4) respond to conventional interferon alpha $($ IFN $-\alpha)$ as poorly as those infected with genotype $1 .{ }^{13} 14$ Pegylated (PEG)-IFN- $\alpha$ plus oral ribavirin became the current standard of care for chronic hepatic $\mathrm{C}$ due to the higher efficacy of PEG-IFN based regimens in inducing improved sustained virological responses (SVRs) compared with IFN- $\alpha$ plus ribavirin. ${ }^{15-17}$ Recent reports showed that the duration of therapy should be individualised according to genotype as patients infected with genotype 1 require treatment for 48 weeks and those with HCV genotypes 2 or 3 seem to be adequately treated for 24 weeks. ${ }^{16-18}$ To date, the efficacy and appropriate duration of PEG-IFN- $\alpha$ and ribavirin therapy have not been evaluated. Therefore, we prospectively compared the effectiveness and safety of 24,36 , or 48 weeks of treatment with PEG-IFN- $\alpha-2 b$ and ribavirin for initial treatment of patients with genotype 4 chronic HCV infection.

\section{PATIENTS AND METHODS}

\section{Patient selection}

Eligible patients included adult males and females with documented chronic hepatitis $\mathrm{C}$ according to the following criteria: elevated serum alanine aminotransferase (ALT) above the upper limit of normal (40 U/l) on two occasions during the preceding six months; anti-HCV positive antibody status assessed by second generation enzyme linked immunosorbent assay (Roche Diagnostics, Branchburg, New

Abbreviations: $\mathrm{HCV}$, hepatitis $\mathrm{C}$ virus; $\mathrm{HCV}-4, \mathrm{HCV}$ genotype 4 ; $\mathrm{HCV}$ 1, HCV genotype 1; PEG-IFN- $\alpha-2 b$, peginterferon alpha- $2 b$; EVR, early virological response; SVR, sustained virological response; ETR, end of treatment response; ALT, alanine aminotransferase; ITT, intention to treat 


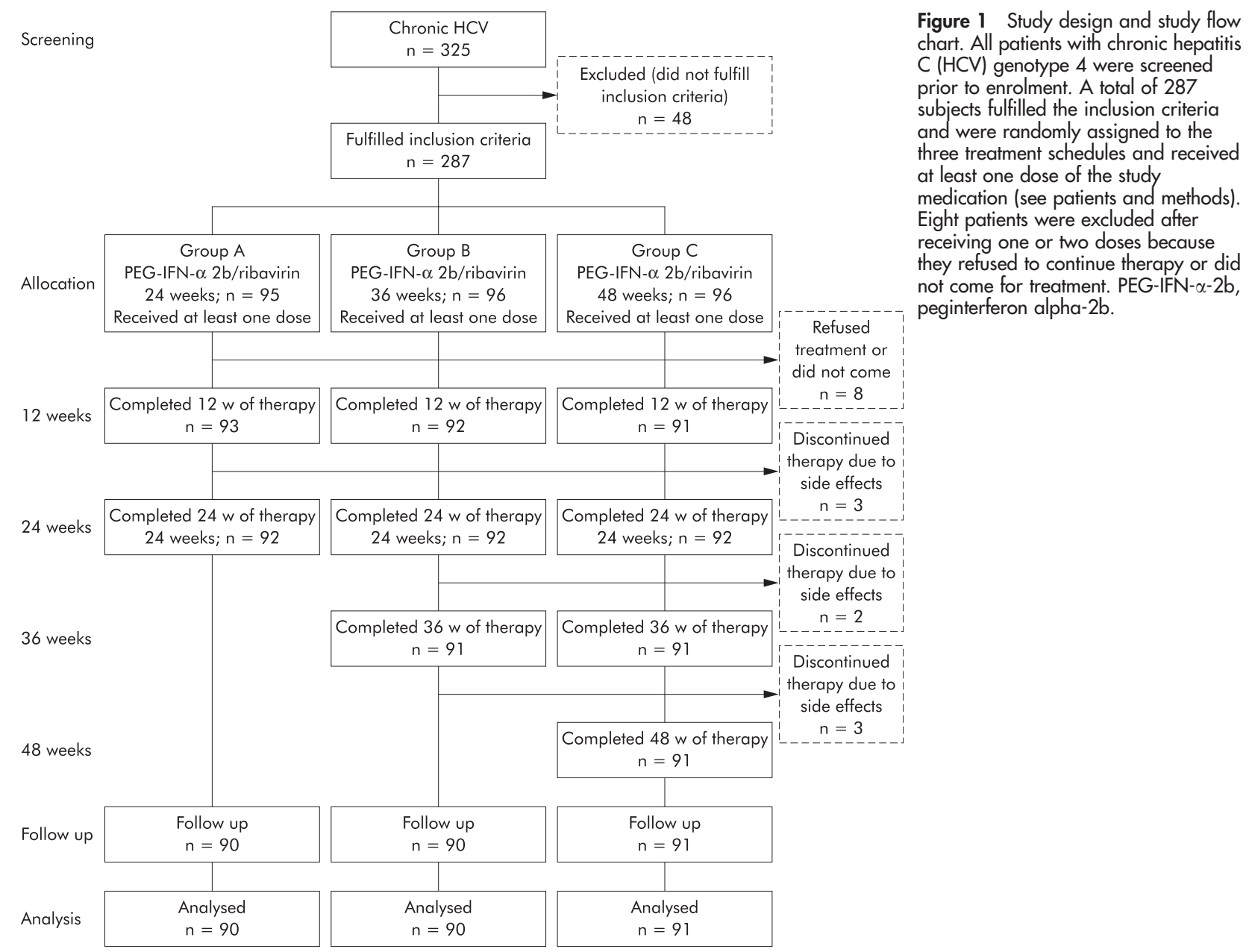

Figure 1 Study design and study flow chart. All patients with chronic hepatitis $\mathrm{C}(\mathrm{HCV})$ genotype 4 were screened prior to enrolment. A total of 287 subjects fulfilled the inclusion criteria and were randomly assigned to the three treatment schedules and received at least one dose of the study medication (see patients and methods). Eight patients were excluded after receiving one or two doses because they refused to continue therapy or did not come for treatment. PEG-IFN- $\alpha-2 b$, peginterferon alpha- $2 b$.

Jersey, USA); positive polymerase chain reaction for HCV RNA (Amplicor HCV Monitor version 1.0; Roche Diagnostics; lower limit of quantitation 2000 copies $/ \mathrm{ml}$ ); genotype 4; and criteria for chronic hepatitis $\mathrm{C}$ in liver biopsy performed within the preceding year with no signs of cirrhosis or bridging fibrosis on pretreatment liver biopsy.

Patients were excluded during screening for any of the following reasons: previous IFN- $\alpha$ therapy; other liver diseases such as hepatitis A, hepatitis B, schistosomiasis, autoimmune hepatitis, alcoholic liver disease, drug induced hepatitis, or decompensated liver disease; coinfection with schistosomiasis or human immunodeficiency virus; neutropenia $\left(<1500 / \mathrm{mm}^{3}\right)$; thrombocytopenia $\left(<90000 / \mathrm{mm}^{3}\right)$; creatinine concentration $>1.5$ times the upper limit of normal; serum $\alpha$ fetoprotein concentration $>25 \mathrm{ng} / \mathrm{ml}$; organ transplant; neoplastic disease; severe cardiac or pulmonary disease; unstable thyroid dysfunction; psychiatric disorder; current pregnancy or breast feeding; or therapy with immunomodulatory agents within the last six months.

\section{Study design}

This was a randomised, double blind, multicentre study comparing the efficacy and safety of 24,36 , or 48 weeks of PEG-IFN- $\alpha$-2b plus ribavirin as initial treatment of patients with proven chronic HCV genotype 4 infection. The study was conducted at four Egyptian centres. Enrolment began in January 2002 and the trial was completed in May 2004. Informed written consent was obtained from each patient. The study protocol was approved by the institutional review boards at each centre and was conducted according to the ethical guidelines of the 1975 Declaration of Helsinki.

The study was conducted using intention to treat (ITT) analyses, defined as inclusion of patients who received at least one dose of any study medication. A total of 335 patients were initially screened; 48 patients were excluded from the study because they did not meet the inclusion criteria (fig 1). Hence 287 patients fulfilled the inclusion criteria and were randomly assigned to receive PEG-IFN- $\alpha-2 b$ (PEG-Intron; Schering Corp., Kenilworth, New Jersey, USA) at a dose of $1.5 \mu \mathrm{g} / \mathrm{kg}$ each week plus ribavirin (Rebetol; Schering Corp.) at a dose $10.6 \mathrm{mg} / \mathrm{kg} /$ day for 24 weeks (group A, $\mathrm{n}=95$ ), 36 weeks (group $\mathrm{B}, \mathrm{n}=96$ ), or 48 weeks (group $\mathrm{C}, \mathrm{n}=96$ ). Compliance was assessed by monitoring empty PEG-IFN- $\alpha-2 b$ vials and used returned syringes. PEGIFN- $\alpha-2 b$ and ribavirin doses were discussed and carefully reviewed with patients at each visit to ensure that the regimens were being followed. All treated subjects were followed for 48 weeks after completion of therapy.

\section{Assessment and end points}

Clinical and biochemical laboratory values (serum ALT, aspartate transaminase, albumin, bilirubin concentrations, and prothrombin time) were determined before initiation of therapy, at entry, at weeks 1, 2, 4, 6, 8, and 12, and monthly thereafter until the end of follow up. HCV genotype was determined with the line probe assay (Inno-LiPA; Innogenetics NV, Zwijnaarde, Belgium). Efficacy was assessed by measuring HCV RNA in serum and serum 
aminotransferases. All subjects who received at least on dose of PEG-IFN- $\alpha-2 b$ plus ribavirin were included in the efficacy analysis.

The primary efficacy end point was SVR (defined as undetectable levels of HCV RNA on analysis with Cobas Amplicor HCV version 2.0, with a lower limit of detection of 100 copies $/ \mathrm{ml}$ at the end of follow up). Secondary end points included virological response over time. Early virological response (EVR) was defined as undetectable HCV RNA serum levels $(<50 \mathrm{IU} / \mathrm{ml})$ or a minimum $2 \log _{10}$ decrease from baseline in HCV RNA at week 12. EVR was correlated with SVR in order to assess its predictive value. End of treatment response (ETR) was defined as undetectable HCV RNA serum levels $(<50 \mathrm{IU} / \mathrm{ml})$ at the end of the scheduled treatment period

\section{Assessment of safety}

Safety was assessed by recording adverse events in the three studied groups, as reported by patients, by clinical and laboratory test results (including haematology, blood chemistry, thyroid function tests, and urinalysis), and by vital data at weeks 1, 2, 4, 6, 8 and 12, monthly thereafter during treatment, and at the end of therapy and follow up. Patients who discontinued therapy prematurely because of intolerance were encouraged to remain in the study. Stepwise reductions in PEG-IFN- $\alpha-2 b$ or ribavirin dose were allowed to manage adverse events or laboratory abnormalities that had reached predetermined thresholds of severity. If the adverse event resolved or improved, a return to initial dosing levels was permitted unless the patient had received the reduced dose for more than four weeks. Patients were withdrawn from treatment if they missed four consecutive doses.

\section{Assessment of HCV kinetic parameters}

Viral decline for all patients was analysed using a mathematical model and methods describing the dynamic relationship between uninfected target cells $(T)$, infected hepatocytes $(I)$, and free virions $(V)$. These models assume rates for clearance of free virus $(c)$, infected cell loss $(\delta)$, virus production $(p)$, and de novo infection $(\beta){ }^{19}$ Early effectiveness of the treatment at inhibiting viral production is modelled by efficiency factors $(\epsilon)$ and $\eta$ on virus production and on de novo infection, respectively, and was assumed to start after a short delay time (t0). A further immunological effect was modelled by an inflation factor $M \geqslant 1$ on infected cell loss, occurring after a delayed time point (tl). Pretreatment infected cell loss $(\delta)$ changes to $M \delta$, which results in a triphasic viral decay. This triphasic model was used to fit data for the first four weeks for individual kinetic parameters, as previously reported. ${ }^{20}$

\section{Liver histology}

Paired liver biopsies were performed for all patients. Liver biopsies were obtained within six months of enrolment and at the end of the post treatment observation period. Liver biopsies were stained with haematoxylin-eosin and a connective tissue stain (chromotrope aniline blue). Liver biopsies were read by two pathologists in a blinded fashion adopting the grading and scoring system proposed by Ishak and colleagues $^{21}$ which includes grading of piecemeal necrosis, confluent necrosis, apoptosis, focal inflammation, and portal inflammation, with a score ranging from 0 to 18 and a staging score ranging from 0 to 6 , with different extents of fibrosis.

\section{Statistical analysis}

Efficacy analyses were performed using an ITT population, defined as all enrolled patients who received at least one dose of any study medication. Two sided 95\% confidence intervals
(CIs) were calculated for each efficacy variable, expressed as per cent of patients. The global hypothesis of no differences among the three treatment groups was tested at a significance level of 0.05; if there was a significant difference among the three treatment groups, each treatment comparison was then tested at a significance level of 0.05. Treatment responses were compared with the use of Fisher's exact test. Predictors of the treatment response were examined by stepwise logistic regression analysis or multivariate Cox regression analysis. For histological responses, scores were calculated before and after treatment, and the changes in scores were compared with the use of the Wilcoxon rank sum test. All reported p values are two sided.

\section{RESULTS}

\section{Baseline characteristics of the patients}

Of 335 patients screened, 48 patients were excluded from the study because they did not meet the inclusion criteria, while 287 patients (150 males and 137 females; mean age 42.0 (9.5) years) fulfilled the inclusion criteria and were randomly assigned to the three treatment schedules and received at least one dose of the study medication (fig 1). Eight patients were excluded after receiving one or two doses because they refused to continue therapy or did not come for treatment. Apart from the eight patients who were excluded early in the study, three patients discontinued therapy before week 24 , and two patients (group $\mathrm{B}, \mathrm{n}=1$; group $\mathrm{C}, \mathrm{n}=1$ ) withdrew after week 24. Another three subjects in group C withdrew after 36 weeks (fig 1). The most common reason for discontinuation of therapy was adverse events in the form of haematological complications, followed by depression, not improved by dose modification. A total of 271 patients (90 subjects in group A, 90 in group B, and 91 in group C) completed the proposed therapy protocol. The three groups were comparable with respect to baseline characteristics (table 1). All patients were infected with HCV genotype 4.

\section{Outcome of therapy}

At the end of treatment (ETR), viral clearance was achieved in $45 / 95$ subjects treated for 24 weeks $(48 \%$ ) versus $65 / 96$ patients $(68 \%)$ treated for 36 weeks and $67 / 96$ patients $(70 \%)$ treated for 48 weeks $(p=0.04$ between groups $A$ and $B$; $\mathrm{p}=0.02$ between groups $\mathrm{A}$ and $\mathrm{C} ; \mathrm{p}=0.4$ between groups $\mathrm{B}$ and C) (fig 2A). SVR (that is, no detectable HCV RNA 48 weeks after completion of therapy) was achieved in 28/95 $(29 \%), 63 / 96(66 \%)$, and 66/96 (69\%) patients treated with PEG-IFN- $\alpha-2 b$ and ribavirin for 24, 36, and 48 weeks, respectively $(p=0.001$ between groups $A$ and $B ; p=0.001$ between groups $\mathrm{A}$ and $\mathrm{C} ; \mathrm{p}=0.5$ between groups $\mathrm{B}$ and $\mathrm{C}$ ) (fig 2A). Significantly more patients treated with long duration PEG-IFN- $\alpha-2$ b plus ribavirin (36 and 48 weeks) had an SVR than those treated with PEG-IFN- $\alpha-2 b$ plus ribavirin for 24 weeks (66\% and 69\% v 29\%; p <0.001) (fig 2A). Virological relapse was highest in patients treated for 24 weeks $(n=20)$ compared with longer treatment periods (relapse: $\mathrm{n}=2$ with 36 week group and $\mathrm{n}=1$ with 48 week group) suggesting that at least 36 weeks of PEGIFN- $\alpha-2 b$ plus ribavirin is required for treatment of patients with chronic hepatitis C genotype 4 to maintain an SVR. Dividing the results further according to pretreatment viral RNA levels showed that among patients with high pretreatment viral RNA levels ( $>2$ million copies $/ \mathrm{ml}$ ) who achieved SVR, $65 \%$ received PEG-IFN- $\alpha-2 b$ plus ribavirin for 48 weeks and $35 \%$ received the 36 week schedule $(p=0.04)$, while none belonged to the 24 week group, suggesting that 48 weeks of therapy might be more appropriate for those with high pretreatment HCV-RNA titres. 
Table 1 Demographic and baseline characteristics of the patients in the three groups who received peginterferon alpha-2b (PEG-IFN- $\alpha-2 b$ ) plus ribavirin for 24,36 , or 48 weeks

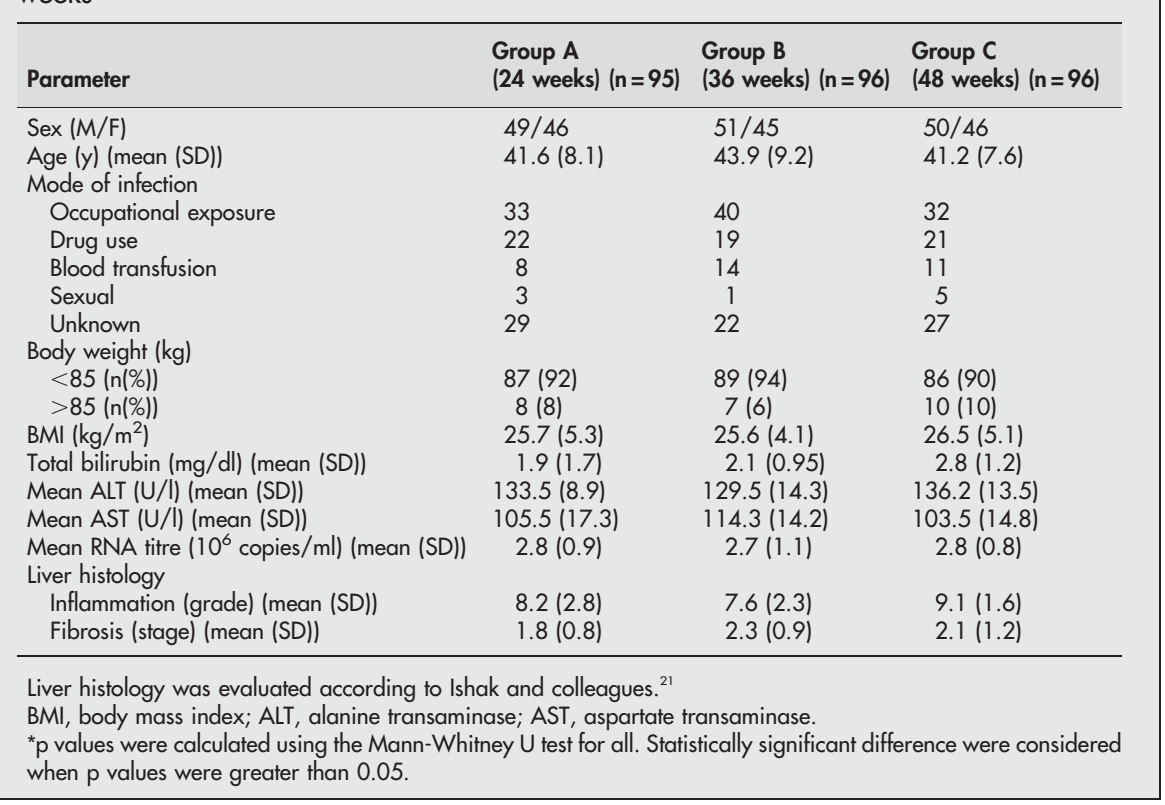

\section{Early virological response}

We examined the significance of undetectable levels of HCV RNA at 12 weeks as a predictor of SVR. An EVR, defined as a reduction in the HCV RNA level by at least 2 log $\mathrm{IU} / \mathrm{ml}$ (on a base 10 scale) at 12 weeks, has been shown in our study to predict SVR, particularly in the 36 and 48 week schedules. The absence of an EVR was not associated with early treatment discontinuation (before week 12) or dose
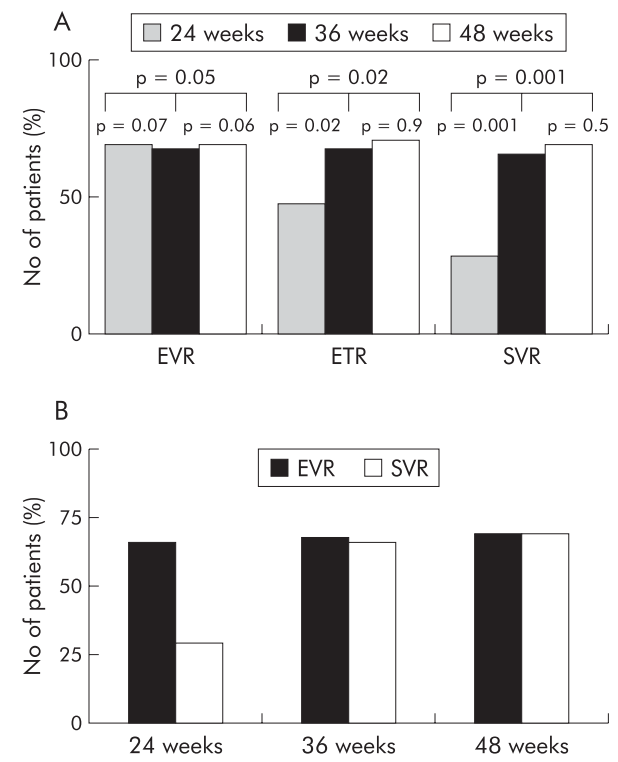

Figure 2 Early virological response (EVR) at weeks 12, end of treatment virological responses (ETR: weeks 24,36 , and 48 after therapy in groups $A, B$, and $C$, respectively), and sustained virological response (SVR: 48 weeks after completion of therapy). (A) Comparison of outcome of treatment in the three patient groups: group $A$, peginterferon alpha-2b (PEG-IFN- $\alpha-2 b)$ plus ribavirin $(n=95)$ for 24 weeks; group B, PEG-IFN- $\alpha$-2b plus ribavirin for 36 weeks $(n=96)$, and PEG-IFN- $\alpha-2 b$ plus ribavirin for 48 weeks $(n=96)$. (B) Percentage of patients achieving early virological response at week 12 and sustained virological response to the different treatment regimen in genotype 4 . modification in the current study (data not shown). In our study, EVR was detected in 199 treated subjects (66 (69\%), 65 $(68 \%)$, and $66(69 \%)$ subjects in groups A, B, C respectively; $\mathrm{p}=0.7$ between groups $\mathrm{A}$ and $\mathrm{B} ; \mathrm{p}=0.5$ between groups $\mathrm{A}$ and $C ; p=0.6$ between groups $B$ and C) (fig 2A). Subjects with an EVR by week 12 were more likely to have an SVR than those who did not .Of those with an EVR $(n=199), 151$ (76\%) subsequently had an SVR. In group A, 25 of 66 (38\%) group A patients with an EVR went on to an SVR compared with $63 / 65(97 \%)$ and $66 / 66(100 \%)$ in groups B and C, respectively $(\mathrm{p}=0.002$ between groups $\mathrm{A}$ and $\mathrm{B} ; \mathrm{p}=0.001$ between groups $A$ and $C ; p=0.8$ between groups $B$ and $C$ ). In all groups, no patient without an EVR ultimately had an SVR. Interestingly, there was a significant difference in SVR rates between short duration of therapy ( 24 weeks; group A) and longer treatment schedules ( 36 weeks, group $\mathrm{B}$ and 48 weeks, group B) despite the finding that the EVR rates showed no significant difference among the three groups. Our results suggest that longer treatment duration is necessary to maintain the virological response. Thus the positive predictive value of an EVR may be adequate only with appropriate treatment duration.

\section{Viral kinetics}

The mean effectiveness of Peg-IFN $\alpha$-2b/ribavirin in blocking viron production was a decline of $88.6 \%$ during the first day of treatment. Early viral kinetics did not show significant differences among the three patient groups either in the estimated kinetic parameter $c$, which determines the first phase decay, or in the estimated pretreatment infected cell loss $\delta$, which determines the second phase decay. The third phase of viral decline started about 7-28 days after therapy at a similar level of viraemia for all treatment groups. The triphasic viral decline pattern was detected in 104 subjects (36\%) (22 in group A, 60 in group B, and 59 in group C) and a biphasic pattern was detected in $183(64 \%)$ patients (table 2 ).

Analysis of the infected cell loss rate, $M \delta$, which determines the third phase decay, probably enhanced by treatment after some delay $(t 1)$, revealed two patterns of response-namely, fast response with very fast decay and undetectable HCV RNA 7-10 days after starting treatment 
Table 2 Baseline clinical, virological and histological, parameters in sustained responders and non-responders in the three patient groups

\begin{tabular}{|c|c|c|c|}
\hline Clinical parameter & $\begin{array}{l}\text { Sustained responders } \\
\text { ( } n=157)\end{array}$ & $\begin{array}{l}\text { Non-responders } \\
(n=130)\end{array}$ & p Value* \\
\hline $\operatorname{Sex}(M / F)$ & $62 / 89$ & $83 / 45$ & $0.07 / 0.04^{*}$ \\
\hline Age (y) (mean (SD)) & $39.6(8.1)$ & $45.2(7.6)$ & 0.07 \\
\hline \multicolumn{4}{|l|}{ Duration of therapy } \\
\hline 24 weeks & 28 & 67 & 0.03 \\
\hline 36 weeks & 63 & 33 & \\
\hline 48 weeks & 66 & 30 & \\
\hline \multicolumn{4}{|c|}{ Risk factors for HCV transmission (n (\%)) } \\
\hline Occupational & $58(38.4)$ & $42(32.8)$ & \\
\hline IV drug use & $30(19.9)$ & $22(17.2)$ & \\
\hline Blood transfusion & 18 (11.9) & 15 (11.7) & \\
\hline Sexual & $7(4.6)$ & $1(0.8)$ & \\
\hline Unknown & $38(25.2)$ & 17 (13.3) & \\
\hline Total bilirubin (mg/dl) & $0.9(2.6)$ & $0.8(0.4)$ & $0.04^{*}$ \\
\hline ALT (IU/I) & $125.3(204.6)$ & $92.5(183.4)$ & 0.95 \\
\hline Viral load $\left(\times 10^{6} \mathrm{cop} / \mathrm{ml}\right)$ & $1.2(0.2)$ & $3(1.9)$ & $0.02^{*}$ \\
\hline Triphasic decay pattern & $94(62.3)$ & $60(46.9)$ & 0.05 \\
\hline Efficiency factor $€(\%)$ & $89.3(4.6)$ & $58.2(5.4)$ & 0.003 \\
\hline Viral clearance $c$ (/day) & $8.5(1.9)$ & $10.6(2.5)$ & 0.2 \\
\hline Death rate $\delta$ (/day) & $0.71(0.3)$ & $0.03(0.01)$ & 0.02 \\
\hline$M \delta$ (/day) & 0.53 & 0.19 & \\
\hline
\end{tabular}

All subjects had comparable baseline clinical virological characteristics.

$\mathrm{HCV}$, hepatitis C virus; ALT, alanine transaminase.

"p values were calculated by the Mann-Whitney $U$ test for all. Statistically significant differences were considered when $p$ values were greater than 0.05 .

with $\mathrm{M} \delta \geqslant 0.25$ (/day) and slow response with flat decline with $\mathrm{M} \delta \leqslant$ than 0.25 (/day) (fig 3). The half life of free virions was estimated at 3.5 hours and that of infected cells from 1.9 hours to over 70 days. Fast response was detected in $48 / 95(52 \%), 50 / 96(53 \%)$, and 51/96(53\%) subjects treated with PEG-IFN- $\alpha-2 b$ and ribavirin for 24,36 , and 48 weeks, respectively $(p=0.9$ between groups $A$ and $B ; p=0.8$ between groups A and C). We found that $47 / 95$ (49\%), 46/ $96(47 \%)$, and 45/96 (47\%) subjects in groups A, B, and C, respectively, were slow (flat) responders $(\mathrm{p}>0.05$ between all groups).

Sustained responders showed significantly greater efficiency factor $(\epsilon)$ on viral production $(89.3(4.6) \%)$ compared with non-responders (58.2 (5.4)\%; $\mathrm{p}=0.003)$.

SVR and EVR were closely associated with a significant increase in the infected cell loss rate, $\mathrm{M} \delta$, reflecting the third
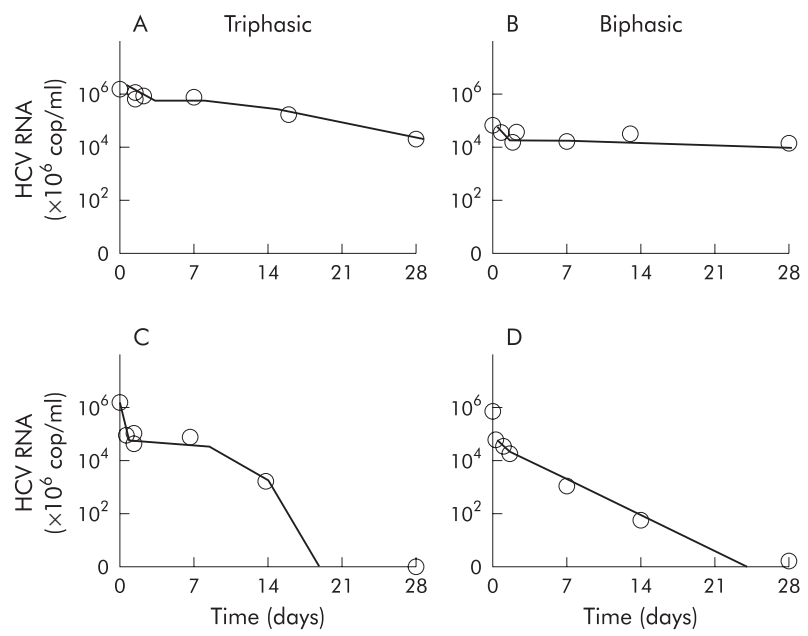

Figure 3 Hepatitis $\mathrm{C}$ virus (HCV) RNA kinetics in chronic hepatitis $\mathrm{C}$ genotype 4 patients treated with peginterferon $\alpha-2 b$ plus ribavirin. Graphs show triphasic or biphasic HCV kinetic patterns in four representative patients: slow responders (A, B) and fast responders (C, D). phase decay $(\mathrm{p}<0.01, \mathrm{p}<0.001$, respectively $)$. We found that $155 / 157$ sustained virological responders (99\%) had M $\delta$ greater than 0.25 (/day) while $\mathrm{M} \delta$ ranged between 0.18 and 0.2 in $2 / 157$ sustained virological responders ( $1 \%)$.

\section{Liver histology}

All patients underwent liver biopsy before and after treatment. Pairwise comparison of histological grading and staging scores for the initial and follow up biopsies showed no deterioration or progression of fibrosis in any patient and improvement $(>2$ point necroinflammatory score improvement) was detected in 155 patients (54\%). Histological response was more likely in those who received longer treatment schedules as histological improvement was detected in 67 patients treated for 36 weeks and in 71 treated for 48 weeks versus 12 treated for 24 weeks. Improvement in histological score was greatest in sustained virological responders who showed a 3-5 point decrease in necroinflammatory (grading) scores and regression in fibrosis scores (fig 4). Fourteen relapsers showed $>2$ point improvement in the grading score in follow up biopsies

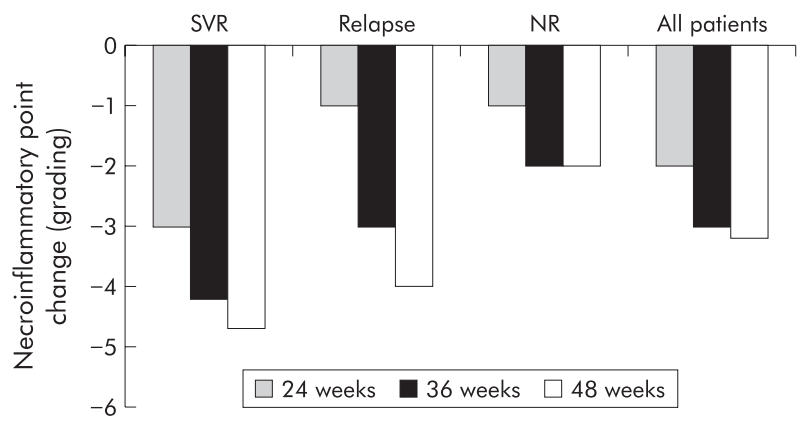

Figure 4 Rates of histological response in the three groups who received peginterferon alpha-2b plus ribavirin for 24,36 , or 48 weeks. Histological response was defined as $\geqslant 2$ point improvement in necroinflammatory score (grading). Histological response in shown for sustained virological responders (SVR), relapsers, non-responders (NR), and all patients. Bars represent change from baseline median total grading score. 
while 12 patients had no substantial changes ( $<2$ points) from the baseline biopsy. The decrease was more obvious for total inflammatory activity and periportal and lobular necrosis than for portal inflammation (data not shown). Comparing baseline characteristics of patients who achieved significant histological response and those who had no histological improvement in the second biopsy identified histological responders as being younger (mean 37 (9) $v 48$ (12) years in those with no improvement), having lower mean pretreatment grading scores (4.3 (1.8) v 9.5 (3.6); $\mathrm{p}=0.02)$ and fibrosis stage $(1.2(1.1) \vee 2.9(1.9) ; \mathrm{p}=0.04)$ The presence of steatosis in the pretreatment liver biopsy did not significantly affect the histological or virological response. The majority of patients who had histological improvement in the follow up biopsy belonged to the groups that had the longer PEG-IFN schedules.

\section{Variables associated with PEG-IFN responsiveness}

To identify predictors of an SVR, the following factors were evaluated: age $(<40$ years $v \geqslant 40$ years $)$, sex, weight $(<75 \mathrm{~kg}$ $v \geqslant 75 \mathrm{~kg}$ or more), mode of HCV transmission, duration of infection ( $<20$ years $v \geqslant 20$ years), rural or urban residence, duration of therapy (24 weeks $v 36 / 48$ weeks), pretreatment ALT quotient $(>3 \quad v \leqslant 3)$, pretreatment HCV RNA level ( 2 million copies $/ \mathrm{ml} v>2$ million copies $/ \mathrm{ml}$ ), and pretreatment histological grading and staging score. Sex, mode of transmission, weight, pretreatment ALT, and histological scores did not predict response to PEG-IFN- $\alpha$-2b/ribavirin therapy. High levels of HCV RNA $(p<0.0001)$ and short treatment period ( 24 weeks, $\mathrm{p}=0.0005$ ) were closely associated with poor response to PEG-IFN- $\alpha-2 b$ plus ribavirin therapy. All patients who relapsed had significantly higher pretreatment HCV-RNA levels compared with sustained responders. Analysis of early viral kinetics showed that rapid response to therapy with greater antiviral efficacy $(\epsilon)>85 \%$, higher rate of infected cell loss $(\delta)>0.5$, and higher viral load decline within the first 24 hours of therapy were associated with response.

\section{Multivariate analysis for factors associated with responsiveness}

The factors identified by multivariate analysis as significant predictors of SVR were viral load $(\leqslant 2$ million copies $/ \mathrm{ml})$ (odds ratio 132.3 (95\% CI 15.3-1043)) and age ( $\leqslant 40$ years) (odds ratio 113.25 (95\% CI 22.09-952.12); p<0.001). Therapy duration was closely related to SVR.

\section{Adverse events}

As shown in table 3, rates and types of adverse events were similar in the three groups. Discontinuation occurred in one $(2 \%)$, two $(2 \%)$, and five $(5 \%)$ patients in groups A, B, and C, respectively (fig 1 , table 3 ) while modification of $1-2$ doses occurred in $14(15 \%), 24(26 \%)$, and $30(32 \%)$ in groups A, B, and $\mathrm{C}$, respectively. The most common events leading to discontinuation were severe haematological abnormalities $(\mathrm{n}=6)$, psychiatric disorders (mainly depression related events; $n=1)$, and hypothyroidism $(n=1)$. Rates of reduction in the dose of PEG-IFN- $\alpha-2 b$ were higher in patients treated for 48 weeks compared with 24 weeks ( $p=0.03$ between groups $\mathrm{A}$ and $\mathrm{C})$ and 36 weeks $(\mathrm{p}=0.04$ between groups $\mathrm{B}$ and $\mathrm{C}$ ).

An initial decrease in median haemoglobin was detected between weeks 1 and 8 in 189 patients. The maximal decrease was greater in patients treated with PEG-IFN- $\alpha-2 b$ plus ribavirin $(4 \mathrm{~g} / \mathrm{dl})$ for 48 weeks. Anaemia improved in 149 patients without dose reduction while dose reduction was required for $2-3$ weeks in $9(9 \%), 13(14 \%)$, and 18 $(19 \%)$ patients in groups A, B, and C, respectively.

Table 3 Adverse events reported by patients in the three groups who received peginterferon alpha- $2 b$ plus ribavirin for 24,36 , or 48 weeks

\begin{tabular}{|c|c|c|c|}
\hline Adverse event & $\begin{array}{l}\text { Group A (24 weeks) } \\
(n=95)\end{array}$ & $\begin{array}{l}\text { Group B (36 weeks) } \\
(\mathrm{n}=96)\end{array}$ & $\begin{array}{l}\text { Group C (48 weeks) } \\
(n=96)\end{array}$ \\
\hline Fatigue & $56(60)$ & $59(64)$ & $62(66)$ \\
\hline Influenza-like illness & $53(57)$ & $58(63)$ & $59(63)$ \\
\hline Headache & $49(53)$ & $52(57)$ & $58(62)$ \\
\hline Myalgia & $48(52)$ & $52(57)$ & $58(62)$ \\
\hline Pyrexia & 41 (44) & $50(54)$ & $53(62)$ \\
\hline Insomnia & $31(33)$ & 35 (38) & $46(49)$ \\
\hline Injection site erythema & $28(30)$ & 34 (37) & $39(42)$ \\
\hline Irritability & $26(28)$ & $33(36)$ & $30(32)$ \\
\hline Back pain & $23(25)$ & $25(27)$ & $29(31)$ \\
\hline Rigors & $16(17)$ & $17(18)$ & $21(22)$ \\
\hline Sore throat & $13(14)$ & 16 (17) & $20(21)$ \\
\hline Cough & $12(13)$ & $15(16)$ & $20(21)$ \\
\hline Pruritus & $10(11)$ & $15(16)$ & $18(19)$ \\
\hline Anorexia & $9(10)$ & $14(15)$ & $18(19)$ \\
\hline Arthralgia & $8(9)$ & $12(13)$ & 17 (18) \\
\hline Dyspnoea & $8(9)$ & 11 (12) & $15(16)$ \\
\hline Rash & $7(8)$ & $10(11)$ & $12(13)$ \\
\hline Depression & $3(3)$ & $3(3)$ & $9(9)$ \\
\hline Dry mouth & $5(5)$ & $7(8)$ & $8(9)$ \\
\hline Alopecia & $4(4)$ & $6(7)$ & 7 (7) \\
\hline Nausea & $4(4)$ & $4(4)$ & 7 (7) \\
\hline Dizziness & $3(3)$ & $5(5)$ & $6(6)$ \\
\hline Abdominal pain & 3 (3) & $5(5)$ & 7 (7) \\
\hline Dry skin & $2(2)$ & $6(7)$ & 7 (7) \\
\hline Diarrhoea & $2(2)$ & $6(7)$ & $8(9)$ \\
\hline Vomiting & $1(2)$ & $3(3)$ & $5(5)$ \\
\hline \multicolumn{4}{|l|}{ Discontinuation of therapy (n (\%)) } \\
\hline Haematological abnormalities & $1(2)$ & $2(2)$ & \\
\hline Psychiatric & 0 & 0 & $3(3)$ \\
\hline Hypothyroidism & 0 & 0 & 1 (1) \\
\hline \multicolumn{4}{|c|}{ Reduction or omission of 1-2 doses for adverse events or laboratory abnormalities } \\
\hline PEG-IFN- $\alpha-2 b$ & $5(5)$ & $11(12)$ & $12(13)$ \\
\hline Ribavirin & $9(10)$ & $13(14)$ & $18(19)$ \\
\hline
\end{tabular}


Haemoglobin levels returned to near baseline values after treatment was completed in all patients. Erythropoietin was not used in this population.

A decrease in median neutrophil counts compared with baseline was reported in all treatment groups at some time point during therapy, particularly during the first eight weeks of treatment. Gradual stabilisation and an increase in neutrophil counts occurred thereafter and counts reached levels comparable with baseline values after completion of therapy. Discontinuation of therapy due to reduction of neutrophil counts below $500 / \mathrm{mm}^{3}$ occurred in one $(1 \%)$, one $(1 \%)$, and three $(3 \%)$ subjects in groups $\mathrm{A}, \mathrm{B}$, and $\mathrm{C}$, respectively (data not shown). Two subjects in group $C$ who discontinued therapy due to neutrophil counts $<500 / \mathrm{mm}^{3}$ developed pneumonia, which was adequately treated. No growth factors were used in this population.

A progressive decrease in median platelet counts was detected in 123 subjects, particularly during the first eight weeks of treatment but gradual stabilisation occurred in 94/ 123 subjects $(76 \%)$. After treatment was completed, median platelet counts returned to normal within four weeks. Three patients with thrombocytopenia (one in group B and two in group C) had a significant decrease in platelet levels in addition to reduction of neutrophil counts with bleeding and thus discontinued treatment.

None of the treated patients had a history of depression or active depression at baseline. Moderate depression (according to Centers for Epidemiological Studies Depression Scale score of 16-20) was reported in three (3\%), three (3\%), and nine $(9 \%)$ patients in groups $\mathrm{A}, \mathrm{B}$, and $\mathrm{C}$, respectively (table 3), and led to discontinuation of therapy in one subject in group C. Initiation of antidepressant medication during treatment in nine patients (two in group A, three in group B, and four in group C) helped continuation of therapy.

Thyroiditis was reported in one patient in group $\mathrm{C}$ which required discontinuation of therapy. There were no unexpected or unusual adverse events reported during this study. None of the responders, relapsers, or non-responders developed decompensation during follow up. No deaths were reported during therapy or follow up.

\section{DISCUSSION}

Previous reports showed that HCV-4 was similarly resistant to conventional interferon monotherapy ${ }^{1422}$ or interferon/ ribavirin combination therapy ${ }^{13}$ as HCV-1. To date, response rates and duration of PEG-IFN-ribavirin treatment in genotype 4 have not been identified and therefore this study investigated the efficacy and safety as well as the duration of PEG-IFN/ribavirin therapy in chronic hepatitis C genotype 4.

We showed that patients chronically infected with HCV-4 responded favourably to PEG-IFN- $\alpha-2 b$ plus ribavirin therapy with SVR rates higher than those reported for genotype 1 with PEG-IFN- $\alpha-2 \mathrm{a} /$ ribavirin $(46-58 \%)$ or PEG-IFN- $\alpha 2 \mathrm{~b} /$ ribavirin $(48 \%)^{15-18}$ but lower than SVR rates in genotypes 2 and $3 .{ }^{15}{ }^{16}$ In the present study, SVR rates of $63 \%$ and $69 \%$ detected in subjects treated for 36 and 48 weeks, respectively, were similar to previous reports from Saudi Arabia involving smaller cohorts infected with genotype $4 .{ }^{23}{ }^{24}$ The high SVR rates in the current study are attributed to selection criteria and close monitoring which increased the compliance of our patients with the prescribed medications, with few patient dropouts and limited dose reduction during the study period. In addition, the study population did not include patients with cirrhosis and only 16 patients were overweight with a body mass index $>30 \mathrm{~kg} / \mathrm{m}^{2}$.

Previous reports showed that treatment with PEG-IFN- $\alpha$ and ribavirin needs to be individualised by genotype as patients with genotype 1 require treatment for 48 weeks while those with genotype 2 or 3 can be adequately treated for 24 weeks. ${ }^{18}$ To date, the appropriate duration of PEGIFN- $\alpha$ /ribavirin therapy for genotype 4 has not been determined and therefore the current study was designed to compare three treatment periods-namely, short treatment for 24 weeks, and longer periods for 36 weeks and 48 weeks. In the current study, significantly higher sustained clearance rates were achieved with the 36 or 48 week schedules compared with the 24 week schedule The significant absolute differences between 24 weeks of treatment and longer treatment periods (48 or 36 weeks) and high virological relapse rate after 24 weeks of treatment suggests that 24 weeks of PEG-IFN- $\alpha-2 b$ /ribavirin therapy is inadequate to treat genotype 4 and, as previously reported in genotype $1,{ }^{18}$ genotype 4 requires longer treatment periods to achieve SVR and decrease virological relapse rates. SVR rates were not significantly different between the 36 and 48 week regimens which may imply that PEG-IFN- $\alpha-2 b$ ribavirin therapy for 36 weeks may be similarly effective as 48 weeks of therapy for HCV-4, although these findings need to be confirmed in larger clinical trials.

To date, the reason for the differences in response of various genotypes to antiviral therapy remains unclear as it is not known why certain genotypes respond more favourably to antiviral therapy than other genotypes. The outcome of therapy may be influenced by a dynamic complex relationship that exists between the pharmacological characteristics of the therapeutic regimen, viral kinetics, and host immune responses. ${ }^{25}$ The "effectiveness" of interferon therapy has been defined as per cent inhibition of virus production during the first day of therapy. ${ }^{20}$ The current study showed that the antiviral effectiveness of PEG-IFN- $\alpha$-2b in blocking production of HCV-4 during the first day was higher than that reported by Halfon and colleagues ${ }^{19}$ during HCV genotype 4 treatment with conventional interferon, which implies a more enhanced antiviral action of PEG-IFN- $\alpha-2 b$ and ribavirin and may explain the greater efficacy of PEG-IFN in inducing SVR rates compared with conventional interferon formulations. The viral dynamics of HCV-4 in our study were more rapid than those detected in patients with chronic hepatitis C genotype 1 treated with PEG-IFN- $\alpha$ but were relatively slower than those of genotypes $2 .{ }^{26}$ The variation in viral dynamic parameters of genotypes during therapy may contribute to the differences in SVR rates and duration of therapy.

EVR to therapy may serve as a decision tool for physicians to identify patients who are unlikely to achieve an SVR and to consider discontinuation of treatment in those patients to spare them the side effects and cost of additional therapy. In the current study, although the EVR rates were not significantly different among the three treatment schedules, SVR rates differed significantly between 24 weeks of therapy and the longer therapy duration (36 weeks and 48 weeks). Pretreatment patient characteristics, patient compliance, and early viral kinetics are unlikely to account for the differences in SVR rates among the short and long treatment schedules as none of these factors differed significantly among the patients groups. In accordance with previous studies, EVR at week 12 in the present study was a reliable clinical time point for prediction of SVR in patients with chronic hepatitis C genotype 4 provided that therapy was completed for at least 36 weeks. Thus our findings suggest that duration of treatment seems to play a crucial role in maintaining EVR and achieving SVR and further confirms several reports showing that PEG-IFN therapy should be individualised according to genotype. ${ }^{18}$

In this study, therapy was not associated with worsening of grading or staging scores in any patient, irrespective of the treatment regimen, as shown in other studies conducted in patients infected with genotypes 1, 2, and 3. ${ }^{15-18} 26$ However, 
in our study, histological improvement in genotype 4 was significantly related to longer duration of therapy, with no significant difference between the 36 week and 48 week regimens. SVR in our study was associated with greater histological improvement in the form of significant necroinflammatory and regression of fibrosis scores, in accordance with other studies in other genotypes ${ }^{25}{ }^{27}$ suggesting that non-cirrhotic patients with an SVR might be regarded as cured. Patients who relapsed benefited from therapy and showed improvement in grading and staging scores in follow up biopsies.

Viral load was highly correlated with PEG-IFN- $\alpha-2 b$ / ribavirin responsiveness, being the best predictive marker for responsiveness, as shown by multivariate analysis in our study and previous studies. ${ }^{15-18}{ }^{23}$ Among patients with high pretreatment viral levels, a substantially higher proportion of those treated with PEG-IFN- $\alpha$-2b plus ribavirin for 48 weeks had an SVR than those treated for 36 weeks while none of those who achieved SVR after the 24 week schedule had high baseline HCV-RNA levels. Pretreatment HCV-RNA levels were not only associated with non-response but were also shown to be associated with an increased risk for virological relapse. Taken together our findings may suggest that traditionally difficult to treat subgroups such as patients with high viral load may benefit more from 48 weeks of therapy.

The commonest adverse events reported in the present study were anaemia and reductions in neutrophil and platelet counts in accordance with previous studies with other genotypes. ${ }^{15-18}$ Although the incidence of adverse events was higher in patients treated with PEG-IFN- $\alpha-2 b$ plus ribavirin for 48 weeks than in those treated for 36 or 24 weeks, these adverse events did not appear to be associated with serious sequelae and were effectively managed by dose reduction or modifications. Short PEG-IFN- $\alpha-2 b /$ ribavirin therapy (24 weeks) was generally better tolerated than the longer regimens but our study showed that 24 weeks of therapy is ineffective in maintaining an SVR in genotype 4.

Taken together, PEG-IFN- $\alpha-2$ b plus ribavirin for 36 weeks was well tolerated and induced reasonable sustained virological and histological responses. The finding that PEG-IFN- $\alpha$-2b plus ribavirin therapy for 36 weeks was better tolerated and similarly effective as 48 weeks of therapy may have important implications for developing countries, particularly in Egypt where chronic hepatitis due to HCV-4 represents an economic burden. Shorter effective therapeutic schemes would provide increased clinical benefit, improve patient compliance and quality of life, in addition to reducing the initial antiviral drug treatment costs and making antiviral treatment more "cost effective". The direct cost attributed to hospital admissions for HCV related complications in the USA was estimated to be $\$ 514$ million in $1995 .{ }^{28}$ In Egypt, the burden of disease would be much greater given that an estimated 12 million ( $20 \%$ of 60 million) are infected with HCV. The probability of patients with chronic HCV developing cirrhosis over a 30 year period ranges from $13 \%$ to $46 \%$ for men and from $1 \%$ to $29 \%$ for women. ${ }^{26}$ In a cross sectional study from Egypt, cirrhosis was detected in 3\% of the study population ${ }^{8}$; even if this value was only $1 \%$, the number of HCV related cirrhosis cases and burden of disease would be significantly greater than that estimated for the USA both now and for the next decade. The incremental cost effectiveness of combination therapy with PEG-IFN ranges from US\$26 000 to 64000 for genotype 1 and from US\$10 000 to 28000 for genotypes 2 and $3 .{ }^{29}{ }^{30}$ Given the additional clinical benefit in the form of higher sustained virological and histological response rates, the improvements in health related quality of life, and expected long term benefit, one may conclude that PEG-IFN and ribavirin therapy may be also cost effective in genotype 4 but this needs to be assessed in a developing country with a high HCV prevalence, such as Egypt.

A limitation of our study was that follow up was restricted to 48 weeks after completion of therapy and thus long term clinical outcomes could not be determined. Late relapse may occur after four years of completion of treatment with conventional interferon and ribavirin according to previous studies. ${ }^{27}$ Long term follow up of sustained virological responders beyond 48 weeks after cessation of therapy is required to determine the long term benefit of therapy. Efficacy, benefit/risk ratio, and cost effectiveness of 36 weeks versus 48 weeks of therapy in HCV-4 require larger trials with longer follow up to identify suitable patients who are most likely to benefit from each treatment schedule. Another limitation is that all of the study subjects had abnormal transaminases and none had cirrhotic changes. None of the subjects had comorbid conditions such as schistosomiasis, which is endemic in Egypt. Thus we do not know whether patients with normal liver enzyme levels, early compensated cirrhosis, or other coexisting conditions will respond similarly.

In conclusion, chronic hepatitis C genotype 4 responds favourably to PEG-IFN- $\alpha$-2b plus ribavirin therapy. We have shown that 48 weeks and 36 weeks of treatment were statistically superior to 24 weeks of treatment. Our observation that 36 weeks of therapy for genotype 4 is well tolerated and induces similar sustained virological response rates to the 48 week regimen may have important clinical implications in the form of increasing adherence, decreasing adverse events, and increasing the cost effectiveness of therapy.

\section{ACKNOWLEDGEMENT}

This study was supported by grants from Fulbright Foundation, NIAID (R21 AI054887), USA, ISID, and by the Alexander von Humboldt Foundation, Bonn, Germany.

\section{Authors' affiliations}

S M Kamal, Department of Gastroenterology and Liver Diseases, Ain Shams Faculty of Medicine, Heliopolis, Cairo, Egypt, and Department of Infectious Diseases of Harvard Medical School and Harvard Institutes of Medicine, Boston, Massachusetts, USA

A A El Tawil, Department of Pathology, Ain Shams Faculty of Medicine, Cairo, Egypt

T Nakano, Division of Viral Hepatitis, Centers for Disease Control and Prevention, Atlanta, Georgia, USA, and Department of Internal Medicine, Ichinomiya Nishi Hospital, Ichinomiya, Aichi, Japan Q He, Department of Infectious Diseases of Harvard Medical School and Harvard Institutes of Medicine, Boston Massachusetts, USA

J Rasenack, Department of Internal Medicine II, Gastroenterology and Hepatology, University of Freiburg, Freiburg, Germany

S A Hakam, W A Saleh, A Ismail, M A Madwar, Department of Gastroenterology and Liver Diseases, Ain Shams Faculty of Medicine, Heliopolis, Cairo, Egypt

A A Aziz, Department of Tropical Medicine, Faculty of Medicine, Ain Shams University, Cairo, Egypt

Conflict of interest: None declared.

\section{REFERENCES}

1 World Health Organization. Global surveillance and control of hepatitis C. Report of a WHO consultation organized in collaboration with the Viral Hepatitis Prevention Board, Antwerp, Belgium. J Viral Hepat 1999;6:35-47.

2 Abdel-Aziz F, Habib M, Mohamed MK, et al. Hepatitis C virus (HCV) infection in a community in the Nile Delta: population description and HCV prevalence. Hepatology 2000;32:111-15.

3 Liang TJ, Rehermann B, Seeff LB, et al. Pathogenesis, natural history, treatment, and prevention of hepatitis C. Ann Intern Med 2000;132:296-305.

4 Alter HJ, Seeff LB. Recovery, persistence, and sequelae in hepatitis $C$ virus infection: a perspective on long-term outcome. Semin Liver Dis 2000;20:17-35.

5 Bhattacheriee V, Prescott LE, Pike I, et al. Use of NS-4 peptides to identify type specific antibody to hepatitis $C$ virus genotypes 1, 2, 3, 4, 5 and 6. J Gen Virol 1995; $76: 1737-48$. 
6 Habib M, Mohamed MK, Abdel-Aziz F, et al. Hepatitis $C$ virus infection in a community in the Nile Delta: risk factors for seropositivity. Hepatology 2001;33:248-53.

7 Kamal S, Madwar M, Bianchi L, et al. Clinical, virological and histopathological features: long-term follow-up in patients with chronic hepatitis C co-infected with S. mansoni. Liver 2000;20:281-90.

8 Angelico M, Renganathan E, Gandin C, et al. Chronic liver disease in the Alexandria governorate, Egypt: contribution of schistosomiasis and hepatitis virus infections. J Hepatol 1997;26:236-43.

9 Morice $Y$, Roulot D, Grando V, et al. Phylogenetic analyses confirm the high prevalence of hepatitis $C$ virus (HCV) type 4 in the Seine-Saint-Denis district (France) and indicate seven different HCV-4 subtypes linked to two different epidemiological patterns. J Gen Virol $2001 ; 82$ (Pt 5): 1001-12.

10 Lyra AC, Ramrakhiani S, Bacon BR, et al. Infection with hepatitis $C$ virus genotype 4 in the United States. J Clin Gastroenterol 2004;38:68-71.

11 Chamberlain R, Adams N, Saeed A, et al. Complete nucleotide sequence of a type 4 hepatitis $C$ virus variant, the predominant genotype in the Middle East. J Gen Virol 2004;78:1341-7.

12 Ray SC, Arthur RR, Carella A, et al. Genetic epidemiology of hepatitis C virus throughout Egypt. J Infect Dis 2000;182:698-707.

13 El-Zayadi A, Selim O, Haddad S, et al. Combination treatment of interferon $\alpha$ $2 \mathrm{~b}$ and ribavirin in comparison to interferon monotherapy in treatment of chronic hepatitis C genotype 4 patients. Ital J Gastroenterol Hepatol 1999:31:472-5.

14 Kamal SM, Madwar MA, Peters T, et al. Interferon therapy in patients with chronic hepatitis C and schistosomiasis. J Hepatol 2000;32:172-4.

15 Fried MW, Shiffman ML, Reddy KR, et al. Peginterferon alfa-2a plus ribavirin for chronic hepatitis C virus infection. N Engl J Med 2002;347:975-82.

16 Manns MP, McHutchison JG, Gordon SC, et al. Peginterferon alfa-2b plus ribavirin compared with interferon alfa- $2 \mathrm{~b}$ plus ribavirin for initial treatment of chronic hepatitis C: a randomised trial. Lancet 2001;358:958-65.

17 Bruno S, Camma C, Di Marco V, et al. Peginterferon alfa-2b plus ribavirin for naive patients with genotype 1 chronic hepatitis $C$ : a randomized controlled trial. $J$ Hepatol 2004;41:474-81.

18 Hadziyannis SJ, Sette H Jr, Morgan TR, et al. PEGASYS International Study Group. Peginterferon-alpha2a and ribavirin combination therapy in chronic hepatitis C: a randomized study of treatment duration and ribavirin dose, Ann Intern Med 2004; 140:346-55.

19 Halfon $\mathrm{P}, \mathrm{Neumann} A U$, Bourliere $M$, et al. Slow viral dynamics of hepatitis $C$ virus genotype 4. J Viral Hepat 2003;10:351-3.

20 Herrmann $\mathrm{E}$, Lee $\mathrm{JH}$, Marinos $\mathrm{G}$, et al. Effect of ribavirin on hepatitis $\mathrm{C}$ viral kinetics in patients treated with pegylated interferon. Hepatology 2003;37:1351-8

21 Ishak K, Baptista A, Bianchi L, et al. Histological grading and staging of chronic hepatitis. J Hepatol 1995;22:696-9.

22 El-Zayadi A, Simmonds P, Dabbous $H$, et al. Response to interferon- $\alpha$ of Egyptian patients infected with hepatitis $C$ virus genotype 4. J Viral Hepat 1996;3:261-4

23 Hasan F, Asker H, Al-Khaldi J, et al. Peginterferon alfa-2b plus ribavirin for the treatment of chronic hepatitis C genotype 4. Am J Gastroenterol 2004:99:1733-7.

24 Shobokshi OA, Serebour FE, Skakni L, et al. Pegylated interferon alfa-2a $(40 \mathrm{kDa})$ as a monotherapy or in combination with ribavirin significantly improves end of treatment response rate in hepatitis $C$ virus genotype 4 chronic active hepatitis patients. Saudi Med J 2003;24(suppl 2):S92.

25 Kamal SM, Fehr J, Roesler B, et al. Peginterferon alone or in combination with ribavirin enhances HCV specific CD4+ T helper 1 responses in patients with chronic hepatitis C. Gastroenterology 2002;123:1070-83.

26 Zeuzem S, Herrmann E, Lee JH, et al. Viral kinetics in patients with chronic hepatitis $C$ treated with standard or peginterferon alpha2a. Gastroenterology $2001 ; 120: 1546-9$.

27 Veldt BJ, Saracco G, Boyer N, et al. Long term clinical outcome of chronic hepatitis $C$ patients with sustained virological response to interferon monotherapy. Gut 2004;53:1504-8.

28 Armstrong GL, Alter MJ, McQuillan GM, et al. The past incidence of hepatitis $C$ virus infection: implications for the future burden of chronic liver disease in the United States. Hepatology 2000;31:777-82.

29 Poynard T. Cost effectiveness of pegylated interferon alpha $2 \mathrm{~b}$ and ribavirin combination in chronic hepatitis C. Gut 2003:52:1532.

30 Salomon JA, Weinstein MC, Hammitt JK, et al. Cost-effectiveness of treatment for chronic hepatitis $C$ infection in an evolving patient population. JAMA 2003;290:228-37 